\title{
Use of complementary and alternative medicine among United States adults: the influences of personality, coping strategies, and social support
}

\author{
Keiko Honda, Ph.D., M.P.H.* and Judith S. Jacobson, Dr.P.H. \\ Department of Epidemiology, Columbia University, New York, NY 10032, USA
}

Available online 26 June 2004

\begin{abstract}
Background. Although patterns of utilization of complementary and alternative medicine (CAM) in the community have begun to be described, few studies have addressed the relationships between dispositional psychological factors and the use of CAM. The aim of this study was to examine the associations between CAM use and personality, coping strategies, and perceived social support in a representative sample of adults in the United States.

Methods. Data were drawn from the Midlife Development in the United States Survey (MIDUS), a representative sample of 3,032 adults aged 25-74 in the US population. We analyzed use of acupuncture, biofeedback, chiropractic, energy healing, exercise/movement therapy, herbal medicine, high-dose megavitamins, homeopathy, hypnosis, imagery techniques, massage, prayer/spiritual practice, relaxation/ mediation, and special diet within the last year. Multiple logistic regression analyses were used to evaluate the association of personality, dispositional coping strategies (primary and secondary control), and perceived social support and strain with CAM use, controlling for sociodemographic factors, medical care access, and physical and mental disorders.

Results. Openness was positively associated with the use of all types of CAM except manipulative body-based methods. Extroversion was inversely correlated with the use of mind-body therapies. Primary control was inversely and secondary control directly correlated with the use of CAM. Perceived friend support was positively associated with the use of mind-body therapies, manipulative body-based methods, and alternative medical systems. Perceived partner strain was positively associated with the use of biologically based therapies, and family strain increased the odds of manipulative body-based methods.

Conclusions. This study is the first to document a significant association between specific domains of personality, coping strategies, and social support, and the use of CAM among adults in the general population. Understanding the relationships between psychological factors and CAM use may help researchers and health care providers to address patients' needs more effectively and to achieve better adherence to treatment recommendations.
\end{abstract}

(C) 2004 The Institute For Cancer Prevention and Elsevier Inc. All rights reserved.

Keywords: Complementary and alternative medicine; Coping style; Psychological; Social support; Personality

\section{Introduction}

Complementary and alternative medicine (CAM) is increasingly accepted in the United States both as treatment for illness and as self-care to promote health and well-being [1-3]. Many mainstream physicians are either referring patients to or practicing CAM modalities, and appear to understand the potential usefulness of CAM [4-6]. However, little is known about the dispositional personal factors

* Corresponding author. Department of Epidemiology, Columbia University, Room 719, 722 West 168th Street, New York, NY 10032. Fax: +1-212-305-9413.

E-mail address: kh2086@columbia.edu (K. Honda). associated with CAM use and CAM choices in the general population. Understanding these associations may facilitate the development of evidence-based CAM and enhance adherence to therapeutic recommendations. The National Center for Complementary and Alternative Medicine (NCCAM) has supported research on how health is related to cognition, personality, and social ties [3], but studies of how CAM use is related to these factors may also be worthwhile.

Clinical observations [7-9] suggest that several psychological factors may be relevant to CAM use: (1) dispositional coping strategies (i.e., optimism and pessimism); (2) congruence between the patient's personal values and beliefs about CAM and the physician's perspective; and 
(3) previous experiences of the patient, family members, and friends with the medical system.

Several investigators [10-13] have quantitatively or qualitatively examined the relationship of CAM use with personality, coping styles, ethnicity/culture, and the influence of family and friends. For example, in a study of cancer patients [13], active coping style and religiousness, but not lack of social support or information, were significantly associated with increased use of CAM. Using the Tellgen Absorption Scale (TAS), another study conducted among clinical and community samples [10] found absorption, ${ }^{1}$ which is known to be positively correlated with the trait of "openness to experience" [14], to be an independent predictor of CAM use. Because most patients make choices about CAM use without guidance from a conventional care provider, their own psychosocial characteristics may play a much greater role in their CAM use than in their conventional medical care.

Three features of previous studies have limited our understanding of the roles of psychological factors in CAM use in the community: (1) studies using convenience samples drawn from treatment settings have had limited generalizability; (2) studies of community-based samples have focused on demographic factors and medical conditions and have not explored psychological factors; and (3) few studies have assessed the predictors of specific types of CAM use.

The aim of this study is to evaluate the association of personality, coping strategies, and perceived social support with CAM use and their relative importance in CAM choices.

\section{Methods}

\section{Sample}

The Midlife Development in the United States Survey (MIDUS) is a nationally representative survey of 4,242 persons aged 25-74 years in the noninstitutionalized civilian population of the 48 coterminous United States [15]. The MIDUS Survey was carried out by the John D. and Catherine T. MacArthur Foundation Network on Successful Midlife Development between January 1995 and January 1996. All respondents completed a 30-min telephone interview and filled out two mailed questionnaires estimated to take a total of about 90 min to complete $(86.8 \%$ conditional response rate in the subsample of telephone respondents). The overall response rate was $60.8 \%$. More details on the MIDUS Survey design, filed procedures, and representativeness are provided elsewhere [15]. The total sample of

\footnotetext{
${ }^{1}$ Absorption refers to the disposition to display episodes of total attention 'during which the available representational apparatus seems to be entirely dedicated to experiencing and modeling the attentional object, be it a landscape, a human being, a sound, a remembered incident, or an aspect of one's self' ([16], p. 274).
}

4,242 subjects (unweighted) corresponds to 3,032 , weighted for selection probabilities and non-response to permit generalizability to the US population on age, gender, race, and education [15].

\section{Measures}

\section{CAM use}

Respondents were asked whether they had received any of the following 14 CAM modalities in the past 12 months: acupuncture, biofeedback, chiropractic, energy healing, exercise/movement therapy, herbal medicine, high-dose megavitamins, homeopathy, hypnosis, imagery techniques, massage, prayer/spiritual practice, relaxation/meditation, special diet. We grouped these practices into the five domains proposed by the NCCAM: alternative medical systems (e.g., acupuncture, homeopathy); body-mind therapies (e.g., biofeedback, hypnosis, relaxation/meditation, imagery techniques, and prayer/spiritual practice); biologically based therapies (e.g., herbal medicine, high-dose megavitamins, special diets); energy therapies (e.g., healing touch, Reiki); and manipulative/body-based methods (e.g., massage therapy, exercise/movement therapies, chiropractic) [3].

\section{Personality traits}

Assessment of personality traits in the MIDUS was based on the "big five" factor model [17], which was tested in a pilot study conducted in 1994 with a probability sample of 1,000 men and women, age 30-70 (574 valid cases were usable for item analysis). Respondents were given a list of adjectives representing aspects of personality and asked to use a four-level Likert-scale to describe how much of the time (all, most, some, or a little) each word described them. The adjectives were interpreted as comprising five traits or scales: Agreeableness (helpful, warm, caring, softhearted, sympathetic) $(\alpha=0.80)$ five-item scale; Openness to experience (creative, imaginative, intelligent, curious, sophisticated, adventurous $)(\alpha=0.77)$ seven-item scale; Conscientiousness [organized, responsible, hardworking, (not) careless] $(\alpha=0.57)$ four-item scale; Extroversion (outgoing, friendly, lively, active, talkative) $(\alpha=0.78)$ five-item scale; and Neuroticism (moody, worrying, nervous, (not) calm) $(\alpha=0.74)$ four-item scale. (The alphas are based on the MIDUS national sample.) For each respondent who provided valid values for at least half the adjectives comprising a trait, the trait was scored as the mean of the responses for that trait.

\section{Primary and secondary control strategies}

The two-process model of primary and secondary control is a conceptualization that proposes two main coping strategies by which people may develop a sense of control [18-20]. Primary control refers to individuals' attempts to make external social and physical or behavioral circumstances conform to their personal needs and desires, whereas secondary control refers to individuals' efforts to adapt 
their cognitive and affective states in response to stressful life events [20]. Respondents were asked to indicate how well each of 14 items described them, using a four-point Likert scale $(1=$ not at all, $4=\mathrm{a}$ lot $)$ [21]. Assessment of control strategies in the MIDUS used a three-factor model developed from a study conducted by Wrosch et al. [21]. Scales included a primary control "persistence in goal striving" $(\alpha=0.77)$ five-item scale, a secondary control "positive reappraisals" $(\alpha=0.78)$ four-item scale; and a secondary control "lowering aspiration" $(\alpha=0.63)$ fiveitem scale. Items were recoded so that higher scores indicated higher primary or secondary control.

\section{Perceived social support and strain}

In the MIDUS, social support and social strain were evaluated as emanating from family members (other than the spouse/partner), friends, and spouse/partner. Supportive network exchanges were measured through four items that were parallel for spouse/partner, family members, and friends. The four items were:

- How much do they (family members, not including your spouse or partner; friends; spouse/partner) understand the way you feel about things?

- How much do they really care about you?

- How much can you rely on them for help if you have a serious problem?

- And, how much can you open up to them if you need to talk about your worries?

Strained network exchanges were also measured through four parallel items that read:

- How often do they criticize you?

- How often do they make too many demands on you?

- How often do they let you down when you are counting on them?

- And, how often do they get on your nerves?

All items were answered on a four-point Likert-type scale (support items: $1=\mathrm{a}$ lot; $4=$ not at all; strain items: $1=$ often, $4=$ never). Items were recoded so that higher scores indicated higher support or strain. Cronbach's alpha scores were: Family support (0.82), Family strain (0.80), Friend support (0.88), Friend strain (0.79), Partner support (0.86), and Partner strain (0.81) [22].

\section{Covariates}

Based on previous studies $[1,2,7,13,23,24]$, certain sociodemographic and health-related factors were hypothesized to predispose to CAM use. Health factors including major depression, panic attacks, generalized anxiety disorder as well as heart-related problems, cancer, and obesity were assessed and included in the regression model. Age, gender, marital status, race/ethnicity, and education were included in the model. Health insurance coverage was also included in the model because it is known to affect health care utilization.

\section{Data analysis}

First, $F$-based tests for independence were conducted to compare the sociodemographic, clinical, and psychosocial (personality, coping, and social support) characteristics of respondents who had and had not used any CAM in the past 12 months. Next, descriptive analyses were used to assess the prevalence of each CAM modality by presence and absence of mental and physical disorders. Finally, multiple logistic regression analyses were used to examine the relationships between psychological factors (personality, control strategies, and perceived social support and strain), and CAM use, controlling for sociodemographics (age, gender, race, education, and marital status), health care access, and physical and mental health conditions (major depression, anxiety disorders, panic disorders, heart-related problems, cancer, and obesity).

All psychological factors were measured on a continuous four-point scale. We calculated the mean by summing the scales and dividing by the number of scales for each factor. Hence, these associations reflect the increase in CAM use associated with every 1-point increase in the mean score for each psychological factor. All results reported here are based on weighted data, adjusted for differential probabilities of selection within households and for differences between the sample distribution and the census population distribution on a range of sociodemographic variables.

\section{Results}

Overall, $54 \%$ of the sample $(n=3,032)$ reported having used any kind of CAM in the past 12 months. Individuals who reported CAM use were more likely to be female, white, or college educated than, but were similar in age and marital status to, those who did not use any CAM (see Table 1). Users of CAM were more likely to report mental disorders (major depression and panic disorders) than nonusers. Users were also more likely than nonusers to report physical disorders, but the associations did not reach statistical significance. Users of CAM were more likely than nonusers to be neurotic, to be open, to receive support from friends, and to experience strain from all social ties measured (see Table 1).

Table 2 shows the percentage of individuals with and without mental and physical disorders who reported using the 14 CAM modalities. Among all respondent groups, the most commonly used CAM modality was prayer/spiritual practice, which was used by about $28 \%$ of all respondents. Among those with no mental or physical disorders $(n=$ 1,540 ), the second most commonly used CAM modality was exercise/movement therapy, and the third was relaxa- 
Table 1

Clinical, sociodemographic, and psychosocial characteristics associated with use of CAM (past 12 months) among adults in the community $(N=3,032)$

\begin{tabular}{|c|c|c|c|}
\hline Characteristic & $\begin{array}{l}\text { No use of CAM } \\
(n=1,392) \text { weighted } \%\end{array}$ & $\begin{array}{l}\text { Use of ANY CAM } \\
(n=1,640) \text { weighted } \%\end{array}$ & $F, P$ value \\
\hline Age [mean (SD)] & $44.8(0.38)$ & $45.8(0.44)$ & ns \\
\hline \multicolumn{4}{|l|}{ Gender } \\
\hline Male & $49.8 \%$ & $37.5 \%$ & \multirow[t]{2}{*}{$F=37.5, P<0.0001$} \\
\hline Female & $50.2 \%$ & $62.5 \%$ & \\
\hline \multicolumn{4}{|l|}{ Education } \\
\hline Less than GED & $17.0 \%$ & $9.6 \%$ & \multirow[t]{5}{*}{$F=16.9, P<0.0001$} \\
\hline High school diploma & $42.2 \%$ & $34.6 \%$ & \\
\hline Some college & $22.5 \%$ & $28.4 \%$ & \\
\hline Bachelor's degree & $11.0 \%$ & $16.1 \%$ & \\
\hline Graduate degree (s) & $7.4 \%$ & $11.3 \%$ & \\
\hline \multicolumn{4}{|l|}{ Race } \\
\hline White & $79.7 \%$ & $84.1 \%$ & \multirow[t]{2}{*}{$F=6.32, P=0.012$} \\
\hline Ethnic & $20.7 \%$ & $15.9 \%$ & \\
\hline \multicolumn{4}{|l|}{ Marital status } \\
\hline Married & $68.7 \%$ & $67.6 \%$ & \multirow[t]{5}{*}{ ns } \\
\hline Separated & $3.4 \%$ & $2.1 \%$ & \\
\hline Divorced & $12.3 \%$ & $13.7 \%$ & \\
\hline Widowed & $5.0 \%$ & $4.3 \%$ & \\
\hline Never married & $10.6 \%$ & $12.4 \%$ & \\
\hline \multicolumn{4}{|l|}{ Mental/emotional disorders } \\
\hline Major depression $($ ref = no) & $10.7 \%$ & $17.4 \%$ & $F=20.5, P<0.0001$ \\
\hline Anxiety disorders (ref = no) & $2.6 \%$ & $3.9 \%$ & ns \\
\hline Panic disorders $(\mathrm{ref}=\mathrm{no})$ & $3.5 \%$ & $10.0 \%$ & $F=38.2, P<0.0001$ \\
\hline \multicolumn{4}{|l|}{ Physical disorders } \\
\hline Heart-related conditions $($ ref $=$ no) & $11.4 \%$ & $13.0 \%$ & ns \\
\hline Cancer $($ ref $=$ no $)$ & $5.9 \%$ & $6.9 \%$ & ns \\
\hline Obese $($ ref $=$ no $)$ & $25.8 \%$ & $26.1 \%$ & ns \\
\hline \multicolumn{4}{|l|}{ Personality traits [mean (SD)] } \\
\hline Agreeableness (continuous) & $3.49(0.01)$ & $3.52(0.01)$ & ns \\
\hline Neuroticism (continuous) & $2.25(0.02)$ & $2.31(0.02)$ & $F=4.49, P<0.05$ \\
\hline Openness (continuous) & $2.98(0.02)$ & $3.05(0.01)$ & $F=7.59, P<0.01$ \\
\hline Extroversion (continuous) & $3.23(0.02)$ & $3.20(0.02)$ & ns \\
\hline Conscientiousness (continuous) & $3.39(0.01)$ & $3.41(0.01)$ & ns \\
\hline \multicolumn{4}{|l|}{ Control strategies [mean (SD)] } \\
\hline Persistence (continuous) & $3.26(0.02)$ & $3.22(0.02)$ & ns \\
\hline Positive reappraisals (continuous) & $3.14(0.02)$ & $3.17(0.02)$ & ns \\
\hline Lowering aspirations (continuous) & $2.63(0.02)$ & $2.55(0.02)$ & $F=6.82, P<0.01$ \\
\hline \multicolumn{4}{|l|}{ Social support and strain [mean (SD)] } \\
\hline Partner support (continuous) & $3.53(0.02)$ & $3.52(0.02)$ & ns \\
\hline Family support (continuous) & $3.40(0.02)$ & $3.41(0.02)$ & ns \\
\hline Friend support (continuous) & $3.14(0.02)$ & $3.28(0.02)$ & $F=22.86, P<0.0001$ \\
\hline Partner strain (continuous) & $2.17(0.02)$ & $2.25(0.02)$ & $F=5.07, P<0.05$ \\
\hline Family strain (continuous) & $2.08(0.02)$ & $2.20(0.02)$ & $F=20.08, P<0.0001$ \\
\hline Friend strain (continuous) & $1.91(0.02)$ & $1.99(0.01)$ & $F=10.54, P<0.01$ \\
\hline
\end{tabular}

tion/meditation. The leading two modalities among individuals with mental disorders $(n=322)$ and with physical disorders $(n=943)$, were similar to those of healthy individuals, but the third most commonly used modality was special diets. Among individuals with both mental and physical disorders $(n=227)$, the second and third most common modalities were special diets and exercise/movement therapy. Those with both mental and physical disorders were more likely than the other respondent groups to use all types of CAM except energy healing. Those with both mental and physical disorders were nearly twice as likely as healthy individuals to use herbal medicine and acupuncture and more than twice as likely to use biofeedback and high-dose megavitamins.
Table 3 presents multiple logistic regression results for models in which the dependent variables were the five domains of CAM use and any CAM use. In the prediction of mind-body intervention use, female gender, more education, and mental disorders were associated with significantly increased likelihood of use. Higher levels of openness were associated with the use of all domains of CAM except manipulative body-based methods. The strongest association was that between openness and energy therapies. Positive reappraisals and friend support were associated with a significantly increased likelihood of using mind-body modalities. Higher levels of extroversion and primary control were associated with reduced likelihood of use of such modalities. Having a physical disorder was associated with the use of 
Table 2

Use of different methods of CAM $(N=3,032)$

\begin{tabular}{|c|c|c|c|c|c|}
\hline \multirow[t]{2}{*}{ Method } & \multicolumn{5}{|c|}{ Use of CAM (weighted \%) } \\
\hline & $\begin{array}{l}\text { No mental and } \\
\text { physical only } \\
(n=1,540)(\%)\end{array}$ & $\begin{array}{l}\text { Mental } \\
\text { disorders only } \\
(n=322)(\%)\end{array}$ & $\begin{array}{l}\text { Physical } \\
\text { disorders only } \\
(n=943)(\%)\end{array}$ & $\begin{array}{l}\text { Both mental and } \\
\text { physical disorders } \\
(n=227)(\%)\end{array}$ & Total \\
\hline \multicolumn{6}{|l|}{ Body/mind therapies } \\
\hline Biofeedback & 0.6 & 0.6 & 0.3 & 1.9 & 0.8 \\
\hline Hypnosis & 1.6 & 1.1 & 1.2 & 1.6 & 1.3 \\
\hline Relaxation/meditation & 13.2 & 12.2 & 8.6 & 19.2 & 13.4 \\
\hline Imagery technique & 2.7 & 2.5 & 1.9 & 3.5 & 2.9 \\
\hline Prayer/spiritual practice & 27.9 & 28.7 & 27.6 & 38.2 & 29.6 \\
\hline \multicolumn{6}{|c|}{ Biologically based therapies } \\
\hline Herbal medicine & 4.9 & 4.9 & 3.6 & 9.7 & 5.3 \\
\hline High-dose megavitamins & 3.6 & 3.9 & 3.0 & 9.5 & 4.2 \\
\hline Special diet & 7.8 & 11.4 & 14.6 & 22.2 & 11.7 \\
\hline \multicolumn{6}{|c|}{ Manipulative/body-based methods } \\
\hline Massage therapy & 7.8 & 7.5 & 5.7 & 12.2 & 8.2 \\
\hline Exercise therapy & 15.5 & 16.3 & 16.5 & 20.2 & 17.5 \\
\hline Chiropractic & 9.4 & 10.1 & 10.5 & 13.6 & 10.9 \\
\hline \multicolumn{6}{|l|}{ Alternative medical system } \\
\hline Acupuncture & 0.8 & 0.9 & 0.9 & 1.6 & 1.1 \\
\hline Homeopathy & 2.1 & 2.0 & 1.6 & 3.5 & 2.4 \\
\hline \multicolumn{6}{|l|}{ Energy therapies } \\
\hline Energy healing & 1.6 & 1.3 & 0.7 & 1.4 & 1.7 \\
\hline
\end{tabular}

biologically based and manipulative body-based therapies, but having a mental disorder was associated with use of body-mind interventions. Partner strain was associated with a significantly increased likelihood of use of biologically based therapies, while primary control was associated with a significantly decreased likelihood of use of such therapies. White respondents were about twice as likely as nonwhites to use manipulative/body-based methods. The use of alternative medical systems and energy therapies were not associated with sociodemographic or health factors but with high levels of openness and secondary control and with low level of primary control.

\section{Discussion}

These results suggest that individual psychological characteristics such as personality, coping, and perceived social support may influence CAM use. Assessment of personality and beliefs may therefore provide insight into CAM-seeking behaviors that may affect clinical and research outcomes. For example, openness to experience appeared to be associated with use of almost all types of CAM. Individuals who are open to experience may be more likely than others to use CAM, even when it is not recommended or appropriate. Extroversion was associated with a low frequency of use of mind-body interventions. Extroverted individuals appeared to favor more concrete or active types of CAM. From a practical point of view, extroverted people may be more likely than others to reject therapeutic recommendations or to be non-adherent to mind-body interventions in trials or treatment.

We hypothesized that coping style might also be associated with CAM choices. Specifically, we hypothesized that those who exercise primary control, which involves modifying the environment, and those who exercise secondary control, which involves modifying the self, might make different choices among CAM modalities. Psychologists differ as to whether coping style is an internal attribute (trait) or a transient state brought about by external life circumstances [25]. However, a study of a large number of normal male and female twins [26] found a strong genetic influence on coping strategies including defense, emotional coping, substitution, and active coping, supporting the notion of coping style itself as a partially heritable trait. On the other hand, some evidence [27] suggests that secondary control strategies become increasingly common in late life. Cross-sectional studies [28,29] also suggest that the predominance of secondary control vs. primary control is associated with culture (e.g., traditional Japanese culture as compared to Western culture). These observations suggest that sense of control is not a fixed aspect of coping style. However, adjusting for age and ethnicity (as a proxy for culture), we observed that those with high levels of primary control were significantly less likely to use all types of CAM, except for manipulative body-based methods, than those with lower levels. On the other hand, secondary control appeared to be associated with use of mind-body 
Table 3

Predictors of any CAM and five domains of CAM use (past 12 months) among adults in the community

\begin{tabular}{|c|c|c|c|}
\hline \multirow[t]{2}{*}{ Predictors } & \multicolumn{3}{|l|}{ Odds ratio $(95 \% \mathrm{CI})$} \\
\hline & $\begin{array}{l}\text { Body/mind } \\
\text { intervention }\end{array}$ & $\begin{array}{l}\text { Biologically based } \\
\text { therapies }\end{array}$ & $\begin{array}{l}\text { Manipulative/body-based } \\
\text { methods }\end{array}$ \\
\hline \multicolumn{4}{|l|}{ Sociodemographic } \\
\hline Age (continuous) & $1.00(0.98-1.01)$ & $1.00(0.99-1.02)$ & $1.01(1.00-1.03)$ \\
\hline Gender $($ ref $=$ male $)$ & $1.84(1.31-2.57)^{* * *}$ & $1.90(1.26-2.86)^{* *}$ & $0.95(0.67-1.34)$ \\
\hline Race (ref $=$ ethnic) & $1.34(0.84-2.15)$ & $1.15(0.66-2.01)$ & $2.02(1.08-3.78)^{*}$ \\
\hline Marital status (ref $=$ nonmarried $)$ & $1.34(0.80-2.23)$ & $0.98(0.54-1.78)$ & $0.85(0.50-1.45)$ \\
\hline Education $(\mathrm{ref}=$ no college $)$ & $1.69(1.22-2.32)^{* *}$ & $1.59(1.08-2.34)^{*}$ & $1.07(0.76-1.49)$ \\
\hline \multicolumn{4}{|l|}{ Healthcare access } \\
\hline Insurance coverage $(\mathrm{ref}=\mathrm{no})$ & $1.04(0.73-1.48)$ & $1.09(0.70-1.71)$ & $1.21(0.81-1.80)$ \\
\hline \multicolumn{4}{|l|}{ Medical comorbidities } \\
\hline Psychiatric disorders $(\mathrm{ref}=\mathrm{no})$ & $1.64(1.09-2.48)^{*}$ & $1.42(0.89-2.26)$ & $1.32(0.84-1.99)$ \\
\hline Physical disorders $(\mathrm{ref}=\mathrm{no})$ & $0.80(0.58-1.09)$ & $1.70(1.17-2.47)^{* *}$ & $1.44(1.04-2.00)^{*}$ \\
\hline \multicolumn{4}{|l|}{ Personality traits } \\
\hline Agreeableness (continuous) & $1.13(0.73-1.74)$ & $1.15(0.72-1.83)$ & $0.85(0.56-1.31)$ \\
\hline Neuroticism (continuous) & $0.92(0.70-1.20)$ & $1.26(0.90-1.76)$ & $1.07(0.81-1.41)$ \\
\hline Openness (continuous) & $1.52(1.06-2.17)^{*}$ & $2.20(1.43-3.39)^{* * *}$ & $1.38(0.95-1.99)$ \\
\hline Extraversion (continuous) & $0.64(0.45-0.93)^{*}$ & $0.94(0.62-1.43)$ & $0.97(0.68-1.40)$ \\
\hline Conscientiousness (continuous) & $0.92(0.64-1.33)$ & $0.71(0.46-1.10)$ & $1.24(0.82-1.86)$ \\
\hline \multicolumn{4}{|l|}{ Control strategies } \\
\hline Persistence (continuous) & $0.59(0.41-0.85)^{* *}$ & $0.56(0.37-0.84)^{* *}$ & $0.94(0.64-1.37)$ \\
\hline Positive reappraisals (continuous) & $1.86(1.34-2.57)^{* * *}$ & $1.52(0.97-2.37)$ & $0.91(0.65-1.29)$ \\
\hline Lowering aspirations (continuous) & $1.00(0.73-1.36)$ & $1.01(0.70-1.45)$ & $0.85(0.61-1.18)$ \\
\hline \multicolumn{4}{|l|}{ Social support and strain } \\
\hline Partner support (continuous) & $1.00(0.73-1.37)$ & $1.38(0.96-1.99)$ & $1.19(0.86-1.64)$ \\
\hline Family support (continuous) & $1.15(0.85-1.55)$ & $0.99(0.69-1.41)$ & $0.99(0.74-1.34)$ \\
\hline Friend support (continuous) & $1.40(1.07-1.82)^{*}$ & $1.05(0.75-1.46)$ & $1.45(1.09-1.92)^{* *}$ \\
\hline Partner strain (continuous) & $1.04(0.78-1.40)$ & $1.51(1.07-2.15)^{*}$ & $0.99(0.71-1.38)$ \\
\hline Family strain (continuous) & $1.34(0.98-1.82)$ & $1.14(0.79-1.64)$ & $1.58(1.13-2.22)^{* *}$ \\
\hline Friend strain (continuous) & $1.16(0.82-1.63)$ & $1.15(0.78-1.68)$ & $0.98(0.70-1.37)$ \\
\hline \multirow[t]{2}{*}{ Predictors } & \multicolumn{3}{|l|}{ Odds ratio $(95 \% \mathrm{CI})$} \\
\hline & Alternative medical systems & Energy therapies & Any CAM \\
\hline \multicolumn{4}{|l|}{ Sociodemographic } \\
\hline Age (continuous) & $1.02(0.98-1.06)$ & $0.99(0.96-1.03)$ & $1.00(0.98-1.01)$ \\
\hline Gender $($ ref $=$ male $)$ & $1.98(0.80-4.89)$ & $1.67(0.32-8.77)$ & $1.61(1.18-2.21)^{* *}$ \\
\hline Race (ref $=$ ethnic) & $0.65(0.25-1.70)$ & $0.85(0.17-4.11)$ & $1.50(0.95-2.37)$ \\
\hline Marital status $($ ref $=$ nonmarried $)$ & $0.50(0.17-1.44)$ & $0.28(0.07-1.19)$ & $1.26(0.78-2.02)$ \\
\hline Education $(\mathrm{ref}=$ no college $)$ & $1.89(0.81-4.39)$ & $4.19(0.97-18.18)$ & $1.57(1.15-2.15)^{* *}$ \\
\hline \multicolumn{4}{|l|}{ Health care access } \\
\hline Insurance coverage $(\mathrm{ref}=\mathrm{no})$ & $0.52(0.28-1.52)$ & $1.09(0.28-4.18)$ & $1.02(0.72-1.44)$ \\
\hline \multicolumn{4}{|l|}{ Medical comorbidities } \\
\hline Psychiatric disorders $(\mathrm{ref}=$ no $)$ & $2.60(0.99-6.79)$ & $1.39(0.30-6.37)$ & $1.70(1.13-2.54)^{* *}$ \\
\hline Physical disorders $(\mathrm{ref}=\mathrm{no})$ & $0.91(0.39-2.09)$ & $0.53(0.12-2.33)$ & $1.13(0.83-1.53)$ \\
\hline \multicolumn{4}{|l|}{ Personality traits } \\
\hline Agreeableness (continuous) & $1.69(0.40-7.18)$ & $2.41(0.34-17.08)$ & $1.06(0.73-1.56)$ \\
\hline Neuroticism (continuous) & $1.30(0.51-3.33)$ & $0.79(0.33-1.90)$ & $0.88(0.69-1.13)$ \\
\hline Openness (continuous) & $3.55(1.33-9.44)^{*}$ & $15.77(2.86-86.87)^{* *}$ & $1.65(1.18-2.31)^{* *}$ \\
\hline Extraversion (continuous) & $0.85(0.31-2.28)$ & $0.96(0.26-3.60)$ & $0.65(0.46-0.91)^{*}$ \\
\hline Conscientiousness (continuous) & $0.90(0.35-2.32)$ & $1.64(0.35-7.79)$ & $0.94(0.65-1.35)$ \\
\hline Predictors & Odds ratio $(95 \% \mathrm{CI})$ & & \\
\hline
\end{tabular}




\begin{tabular}{|c|c|c|c|}
\hline & Alternative medical systems & Energy therapies & Any CAM \\
\hline \multicolumn{4}{|l|}{ Control strategies } \\
\hline Persistence (continuous) & $0.24(0.11-0.51)^{* * *}$ & $0.18(0.03-0.93)^{*}$ & $0.67(0.47-0.95)^{*}$ \\
\hline Positive reappraisals (continuous) & $3.41(1.19-9.34)^{*}$ & $3.07(0.77-12.33)$ & $1.20(0.89-1.62)$ \\
\hline Lowering aspirations (continuous) & $0.90(0.48-1.70)$ & $2.62(1.01-6.77)^{*}$ & $0.86(0.64-1.16)$ \\
\hline \multicolumn{4}{|l|}{ Social support and strain } \\
\hline Partner support (continuous) & $1.11(0.54-2.28)$ & $1.41(0.44-4.45)$ & $1.11(0.82-1.49)$ \\
\hline Family support (continuous) & $0.86(0.46-2.00)$ & $0.71(0.22-2.26)$ & $1.27(0.96-1.69)$ \\
\hline Friend support (continuous) & $2.81(1.23-6.41)^{*}$ & $3.53(0.95-13.12)$ & $1.37(1.07-1.74)^{*}$ \\
\hline Partner strain (continuous) & $1.17(0.61-2.25)$ & $0.72(0.29-1.78)$ & $1.11(0.84-1.46)$ \\
\hline Family strain (continuous) & $0.89(0.51-1.58)$ & $1.90(0.78-4.62)$ & $1.54(1.15-2.06)^{* *}$ \\
\hline Friend strain (continuous) & $1.33(0.62-2.85)$ & $0.26(0.06-1.11)$ & $1.15(0.82-1.59)$ \\
\hline
\end{tabular}

interventions and alternative medical systems. Assessing individual differences in coping strategies may help us understand how to tailor patient education.

The effects of social support or strain from friends, family, and partner on the use of CAM are important because they are amenable to psychological interventions. Although these social support measures are self-reported and may not be a true reflection of social support received, we found that social support from friends was associated with most CAM modalities such as mind-body interventions, manipulative bodybased methods, and alternative medical systems. Future research could further examine the structural aspects (e.g., size and kind) of friend network and actual support exchanges (e.g., informational, decisional) in relation to CAM use.

Partner strain and family strain were associated with increased use of biologically based therapies and manipulative body-based methods, respectively. Although the reasons for these associations are beyond the scope of this study, it is possible that some individuals respond to familial strain by using certain types of CAM modalities. Future research is needed on the pathways that link social strain, potentially comorbid psychological distress, personal resources, and CAM use.

This study has several limitations. Our theoretical model proposes that individuals' trait- and cognitive-oriented characteristics affect their CAM use. The cross-sectional design, however, does not allow us to assess causality. For example, as a result of using mind/body CAM, individuals may become more tolerant of a difficult situation and may therefore appear in the MIDUS data to have high secondary control. Similarly, using CAM without adverse effects, at least in the short term, may encourage greater openness to experience. However, personality traits are believed to develop early in life and to remain relatively stable over a person's life span [30,31]. The questions in the MIDUS instrument focus on CAM use during the past 12 months. We therefore doubt that CAM use is the causal agent in most of the associations we observed. However, longitudinal research is needed to test the hypothesis that aspects of personality are predictors of CAM choices. Another limitation is that the survey instrument was not specifically designed to assess determinants of CAM use. For example, specific mental and physical disorders that the MIDUS instrument does not measure may also have played a role in CAM use. The survey used a dichotomous measure of CAM use. Data on dose or frequency of CAM use might shed additional light on CAM seeking behavior. The MIDUS sample included only individuals aged 25-74 years. Some studies have found that older people are less likely to use CAM than younger people. We found no association of CAM use with age, but we cannot generalize our findings to very old or very young adults.

Despite these limitations, the current study has identified direct relationships between trait-oriented and cognitiveoriented characteristics and CAM choices in a sample of the general population. If these factors do affect CAM choices, they may also predict adherence to and outcomes of treatment recommendations in general. Psychosocial data might help providers and patients select treatments for their compatibility with a patient's psychosocial profile. Even now, psychosocial testing is sometimes used to assess the eligibility of candidates for participation in clinical trials. It may be also have the potential to provide not just a general judgment of emotional stability but also an assessment of the fit between the intervention and the subject. In studies of treatments that are expected not to have dramatic short-term effects, small variations in adherence may have important consequences for the interpretability of results. We therefore suggest that future trials, especially in but not limited to CAM, incorporate measurements of psychosocial factors and evaluate them as predictors of adherence.

\section{Key points}

Individual psychosocial characteristics such as personality, coping, and perceived social support may influence CAM use.

\section{Policy implications}

Assessment of personality and coping style has the potential to provide not just a general judgment of emotional 
stability but also an assessment of the fit between the intervention and the subject.

\section{Acknowledgments}

$\mathrm{KH}$ is supported by a postdoctoral fellowship from the National Cancer Institute (CA09529).

\section{References}

[1] Gordon NP, Lin TY. Use of complementary and alternative medicine by the adult membership of a large northern California health maintenance organization, 1999. J Ambul Care Manage 2004;27:12-24

[2] Ni H, Simile C, Hardy AM. Utilization of complementary and alternative medicine by United States adults. Med Care 2002;40:353-8.

[3] Anonymous. General information about CAM and the NCCAM. National Institute of Health. Available at: http://nccam.nih.gov/nccam/ an/general/index.html. Accessed Feb 14, 2004.

[4] Astin JA, Marie A, Pelletier KR, Hansen E, Haskell WL. A review of the incorporation of complementary and alternative medicine by mainstream physicians. Arch Intern Med 1998;158:2303-10.

[5] Berman BM, Singh BB, Hartnoll SM, Singh BK, Reilly D. Primary care physicians and complementary-alternative medicine: training, attitudes, and practice patterns. J Am Board Fam Pract 1998;11:272-81.

[6] Hall J, Bulik R, Sierpina V. Community preceptors' attitudes toward and practices of complementary and alternative medicine: a Texas survey. Tex Med 2003;99:50-3.

[7] Mackenzie ER, Taylor L, Bloom BS, Hufford DJ, Johnson JC. Ethnic minority use of complementary and alternative medicine (CAM): a national probability of CAM utilizers. Altern Ther Health Med 2003;9:50-6.

[8] Ritvo P, Irvine J, Katz J, Matthew A, Sacamano J, Shaw BF. The patient's motivation in seeking complementary therapies. Patient Educ Couns 1999;38:161-5.

[9] Truant T, Bottorff JL. Decision making related to complementary therapies: a process of regarding regaining control. Patient Educ Couns 1999;38:131-42.

[10] Owen JE, Taylor AG, Degood D. Complementary and alternative medicine and psychologic factors: toward an individual differences model of complementary and alternative medicine use and outcomes. J Altern Complement Med 1999;5:529-41.

[11] Maskarinec G, Shumay DM, Kakai H, Gotay CC. Ethnic differences in complementary and alternative medicine use among cancer patients. J Altern Complement Med 2000;6:531-8.

[12] Boon H, Brown JB, Gavin A, Kennard MA, Stewart M. Breast cancer survivors' perception of complementary/alternative medicine (CAM): making the decision to use or not to use. Qual Health Res 1999;9:639-53

[13] Söllner W, Maislinger S, DeVries A, Rumpold G, Lukas P. Use of complementary and alternative medicine by cancer patients is not associated with perceived distress or poor compliance with standard treatment but with active coping behavior. Cancer 2000;89:873-80

[14] Radtke HL, Stam HJ. The relationship between absorption, openness to experience, anhedonia, and susceptibility. Int J Clin Exp Hypn 1991;39:39-56.

[15] Kessler RC, DuPont RL, Berglund P, Wittchen HU. Impairment in pure and comorbid generalized anxiety disorder and major depression at 12 months in 2 national surveys. Am J Psychiatry 1999; $156: 1915-23$.

[16] Tellegen A, Atkinson G. Openness to absorbing and self-altering experiences ("absorption"), a trait related to hypnotic susceptibility. J Abnorm Psychology 1974;83:268-77.

[17] Costa PT, McCrae RR. Revised NEO Personality Inventory (NEO-PIR) and NEO Five Factor Inventory (NEO-FFI): Professional Manual. Odessa, FL: Psychological Assessment Resources, 1992.

[18] Heckhausen J, Schluz R. Optimisation by selection and compensation: balancing primary and secondary control in life-span development. Int J Behav Dev 1993;16:287-303.

[19] Heckhausen J, Schluz R. A life-span theory of control. Psychol Rev 1995;102:284-304.

[20] Rothbaum F, Weisz JR, Snyder SS. Changing the world and changing the self: a two-process model of perceived control. J Pers Soc Psychol 1982;42:5-37.

[21] Wrosch C, Heckhausen J, Lachman ME. Primary and secondary control strategies for managing health and financial stress across adulthood. Psychol Aging 2000;15:387-99.

[22] Walen HR, Lachman ME. Social support and strain from partner, family, and friends: costs and benefits for men and women in adulthood. J Soc Pers Relationsh 2000;17:5-30.

[23] Cuellar N, Aycock T, Cahill B, Ford J. Complementary and alternative medicine (CAM) use by African American (AA) and Caucasian American (CA) older adults in a rural setting: a descriptive, comparative study. BMC Complement. Altern Med 2003;3:8.

[24] Bausel BR, Lee WL, Berman BM. Demographic and health-related correlates of visits to complementary and alternative medical providers. Med Care 2001;39:90-6.

[25] Beutler LE, Moos RH. Coping and coping styles in personality and treatment planning: introduction to the special series. J Clin Psychol 2003;59:1045-7.

[26] Busjahn A, Faulhaber HD, Freier K, Luft FC. Genetic and environmental influences on coping styles: a twin study. Psychosom Med 1999;61:469-75

[27] Chipperfield JG, Perry RP, Menec VH. Primary and secondary control-enhancing strategies: implications for health in later life. J Aging Health 1999;11:517-39.

[28] Weisz JR, Rothbaum FM, Blackman TC. Standing out and standing in: the psychology of control in America and Japan. Am Psychol 1984;39:955-69.

[29] Azuma H. Secondary control as a heterogeneous category. Am Psychol 1984;39:970-1.

[30] Costa PT, McCrae RR. Personality in adulthood: a six-year longitudinal study of self-reports and spouse ratings on the NEO Personality Inventory. J Pers Soc Psychol 1998;54:853-63.

[31] Roberts BW, DelVecchio WF. The rank-order consistency of personality traits from childhood to old age: a quantitative review of longitudinal studies. Psychol Bull 2000;126:3-25. 